\title{
MicroRNA-199a-5p Induced Autophagy and Inhibits the Pathogenesis of Ankylosing Spondylitis by Modulating the mTOR Signaling via Directly Targeting Ras Homolog Enriched in Brain (Rheb)
}

\author{
Yahan Wang Jianping Luo Xiaogang Wang Bin Yang Liyang Cui \\ Department of Orthopedics, Henan Provincial People's Hospital, Zhengzhou, China
}

\section{Key Words}

MicroRNA-199a-5p - Autophagy - Ankylosing spondylitis - Ras homolog enriched in brain (Rheb) • Mechanistic target of rapamycin (mTOR)

\begin{abstract}
Background/Aims: Ankylosing spondylitis (AS) is an inflammatory and immune disease leading to disability. Autophagy has been identified as a potential player in understanding the pathogenesis of AS. Methods: MiRNA-199a-5p and autophagy-related gene expression were determined by qRT-PCR or Western blot. Cytokine production was determined using ELISA assays. Proliferation was determined by MTT assay. MiRNA-199a-5p and Ras homolog enriched in brain (Rheb) were upregulated or downregulated by overexpression of plasmid or siRNA transfection. Results: Expression of miRNA-199a-5p, and autophagy-related genes LC3, beclin1, and ATG5 was significantly decreased in T cells of AS patients. Serum concentrations of TNF- $\alpha$, IL-17, and IL-23 were promoted in AS patients, compared to healthy controls. MiRNA-199a-5p expression levels also showed significant negative correlations with the Ankylosing Spondylitis Disease Activity Score (ASDAS) and modified Stoke Ankylosing Spon dylitis Spinal Score (mSASSS) of AS patients. In Jurkat T cells and T cells isolated from AS patients, miRNA-199a-5p overexpression promoted autophagy-related genes expression and decreased TNF- $\alpha$, IL-17, and IL-23 levels, whereas inhibition of miRNA-199a-5p attenuated these effects. As a direct target of miRNA-199a-5p, Rheb inhibition led to a striking decrease in the phosphorylation of the mechanistic target of rapamycin (mTOR) and induced autophagy. Moreover, pcDNA3.1-Rheb effectively reduced the inhibiting effects of mTOR signaling caused by miRNA-199a-5p overexpression. All effects were offset by pretreating with rapamycin (an mTOR antagonist). Conclusions: AS patients with advanced spinal damage had decreased autophagy levels and that miRNA-199a-5p may induce autophagy and inhibit the pathogenesis of AS by modulating the mTOR signaling via direct targeting Rheb.
\end{abstract}




\section{Cellular Physiology Cell Physiol Biochem 2017;42:2481-2491 \begin{tabular}{l|l} 
DOI: 10.1159/000480211 22, 2017 & $\begin{array}{l}\text { O } 2017 \text { The Author(s). Published by S. Karger AG, Basel } \\
\text { www.karger.com/cpb }\end{array}$ \\
\hline and Biochemistryed onne: August 22,
\end{tabular} \\ Wang et al.: MicroRNA-199a-5p Inhibits Ankylosing Spondylitis Pathogenesis}

\section{Introduction}

Ankylosing spondylitis (AS) is an inflammatory and immune disease that mainly affects young men and causes significant disability [1, 2]. AS manifests predominantly with stiffness, pain, and spinal joint inflammation [3]. In China, AS prevalence is about $0.20 \%$ to $0.40 \%$, almost $80 \%$ of AS patients are young adults, most of whom are male, and the disability rate five years after onset of symptoms is $40 \%$ to $60 \%$ [4-7]. The pathogenesis of AS is not well known, and effective treatment is limited [8]. Autophagy has been studied in AS pathogenesis. From yeast to mammals, autophagy is essential for cellular homeostasis by controlling the digestion of damaged organelles within a cell [9]. Accumulating evidence has indicated that failure of autophagy results in increased reactive oxygen species production and abnormal gene expression, leading to neurodegeneration, cardiomyopathy, abnormal skeletal development, and premature death $[10,11]$. Reports show that in the inflamed gut of AS patients, autophagy was significantly up-regulated [12]. In peripheral blood mononuclear cells (PBMCs) isolated from AS patients, the autophagy-related genes ATG16L1, IRGM, and HSP90AA1 were down-regulated, compared to healthy controls [13].

MicroRNAs (miRNAs) are a large family of non-coding small RNAs composed of 1825 nucleotides. MiRNAs post-transcriptionally regulate the degradation and translation of their target genes. MiRNAs are involved in the inflammatory response and the development of autoimmune diseases, such as rheumatoid arthritis [14], and multiple sclerosis [15]. Increasing evidence has shown that aberrant miRNA expression is linked to the regulation of autophagy [16]. Studies have shown that miR-150, miR-342-5p, miR-16, miR-221, miR$99 b$, let-7b, let-7i, and miR-513-5p were higher and that miR-218, miR-30e, miR-199a-5p, miR-409-3p, and miR-215 were lower in the T cells of AS than in healthy counterparts [17]. MiR-199a-5p, a recently discovered miR, has been reported to be dynamically regulated in the T cells of AS patients, suggesting that miR-199a-5p may play a role in the development of AS. However, the function and downstream molecular pathways of miR-199a-5p during the pathogenesis of AS are unclear.

In the present study, we aimed to investigate whether miR-199a-5p takes part in the pathogenesis of AS and whether it affects autophagy. The underlying mechanism was also explored. These findings provide hope that pharmacologic activation of autophagy or the manipulation of key autophagic machinery-related molecules will lead to new approaches to managing AS.

\section{Materials and Methods}

\section{Antibodies and reagents}

All substances were purchased from Gibco (Grand Island, NY, USA), unless otherwise stated. The antibodies against microtubule-associated protein light chain 3 (LC3)-I/II, beclin1, ATG5, ATG1 and mTOR were obtained from Cell Signaling Technology (Beverly, MA, USA). The antibody to $\beta$-actin was from Anbo Biotechnology Company (San Francisco, CA, USA). Horseradish peroxidase (HRP)-conjugated goat antimouse and anti-rabbit antibodies were from Abcam (Cambridge, MA, USA). Lipofectamine 2000 was purchased from Invitrogen Life Technologies (Carlsbad, CA, USA). Rapamycin was obtained from Sigma Chemical Co. (St. Louis, MO, USA).

\section{Study population}

All samples were obtained from patients at Henan Province Hospital of Traditional Chinese Medicine. Blood samples were obtained from 41 patients with AS (all HLA-B27-positive; 30 males and 11 females; mean age $33.7 \pm 12.4$ years) according to the modified 1984 New York criteria for AS diagnosis [18]. None of these patients received tumor necrosis factor inhibitor treatment. Control blood samples were collected from 36 healthy blood donors (20 male and 16 females; mean age $31.4 \pm 10.2$ years) at Henan Province Hospital of Traditional Chinese Medicine in China who were undergoing routine health examinations and who had no history of autoimmune diseases. All subjects were from the Chinese Han population and were 


\section{Cellular Physiology Cell Physiol Biochem 2017;42:2481-2491 \begin{tabular}{l|l} 
DOI: 10.1159/000480211 22, 2017 & $\begin{array}{l}\text { O 2017 The Author(s). Published by S. Karger AG, Basel } \\
\text { www.karger.com/cpb }\end{array}$ \\
\hline
\end{tabular} \\ Wang et al.: MicroRNA-199a-5p Inhibits Ankylosing Spondylitis Pathogenesis}

recruited because they shared similar genetic and environmental backgrounds with the patient population. At the time of blood collection, an Ankylosing Spondylitis Disease Activity Score (ASDAS) was determined to assess disease activity of AS and the modified Stoke AS Spinal Score (mSASSS) was scored to assess radiographic severity. Two experienced radiologists calculated the mSASSS using cervical and lumbar spine radiographs. This study was approved by the Ethical Committee of Henan Province Hospital of Traditional Chinese Medicine, China. All patients and control subjects provided written informed consent to participate after a full explanation of the study.

T cell isolation and culture

Blood samples were collected into tubes containing EDTA. Ficoll-Hypaque density gradient centrifugation was used to isolate PBMCs at $300 \mathrm{~g}$ and $4^{\circ} \mathrm{C}$ for $10 \mathrm{~min}$. Then magnetic beads coated with anti-human CD3 (Miltenyi Biotec, GmbH, Gladbach, Germany) was used to purify the T cells. The purified T cells were then cultured in Roswell Park Memorial Institute (RPMI) 1640 medium at $37^{\circ} \mathrm{C}$ in $5 \% \mathrm{CO}_{2}$ with $10 \%$ fetal bovine serum, $100 \mathrm{U} / \mathrm{mL}$ penicillin, and $100 \mathrm{U} / \mathrm{mL}$ streptomycin.

The human Jurkat T cell line (ATCC, Manassas, VA, USA) was cultured in the same condition as the isolated T cells.

\section{RNA extraction and quantitative RT-PCR}

Total RNA was extracted from cells using the RNeasy mini kit (Qiagen, Gaithersburg, MD, USA) according to the manufacturer's recommendations. RNA was homogenized using Qiashredder columns (Qiagen). The obtained RNA pellet was dissolved in $30 \mu \mathrm{l}$ of RNase-free water and stored at $-80^{\circ} \mathrm{C}$. Each sample had approximately $5 \mu \mathrm{g}$ of total RNA as determined by spectrophotometry. RNA was reverse transcribed into complimentary DNA (cDNA) using a SuperScript III synthesis kit (Invitrogen) in accordance with the manufacturer's instructions. Gene expression was analyzed by TaqMan Master Mix (Applied Biosystems, Foster City, CA, USA) on an ABI 7300 Real Time PCR System. The specific sequences of primers are as follows (from 5' to 3'): miR-199a-5p, ACA CTC CAG CTG GGC CCA GTG TTC AGA CTA C; LC3, 5'-AGG ATC CAT GCC CTC AGA CCG GCC TTT-3', 5'-CGC TCT AGA TCA GAA GCC GAA GGT TTC CT-3'; beclin1,5'- CGG GAT CCA TGG AAG GGT CTA AGA CGT CC-3', 5'-CGG AAT TCT CAT TTG TTA TAA AAT TGT GAG G-3'; ATG5, 5'-GCG GAT CCA TGA CAG ATG ACA AAG ATG TGC TTC G-3', 5'-ATT AAG CTT TCA ATC TGT TGG CTG GGG CAC AAT G-3'; U6, 5'-CGC AAG GAT GAC AC GCA AAT TC-3'; and $\beta$-actin, 5'-CTG GAA CGG TGA AGG TGA CA-3', 5'-AAG GGA CTT CCT GTA ACA ATG CA-3'. The $\beta$-actin and U6 SnRNA were used as a quantitative and qualitative control, respectively to normalize gene and miRNA expression. Data were analyzed using the formula: $R=2-(\Delta C$ t sample $-\Delta C$ t control $)$.

\section{Western blot analysis}

The whole-cell lysates were obtained using RIPA lysis buffer. Equal amounts of protein from each sample were separated using SDS-PAGE and electro-transferred onto a polyvinylidene difluoride membrane (Roche Diagnostics, Indianapolis, IN, USA). After blocking, blots were probed with primary antibodies against LC3-I/II (1:1000), beclin1 (1:1000), ATG5 (1:1000), ATG1 (1:1000), mTORC1 (1:2000) and $\beta$-actin (1:5000) overnight at $4^{\circ} \mathrm{C}$, followed by HRP-conjugated secondary antibody for $30 \mathrm{~min}$ at $37^{\circ} \mathrm{C}$. Protein bands were visualized using an enhanced chemiluminescence (ECL) kit, quantified using the Image J program, and normalized by $\beta$-actin.

\section{Measurement of serum cytokine concentrations}

Serum concentrations of TNF- $\alpha$, IL-17, and IL-23 were determined by using commercially available enzyme-linked immunosorbent assay (ELISA) kits (R\&D Systems Inc., Minneapolis, MN, USA) according to the manufacturer's protocol. All samples were measured in duplicate.

\section{Oligonucleotide and siRNA transfection}

Jurkat T cells were transfected with miR-199a-5p mimics, miR-199a-5p inhibitors, or miR controls (50 nM, Shanghai GenePharma Co.,Ltd) by using Lipofectamine for $48 \mathrm{~h}$. RhebsiRNA transfection was performed according to the manufacturer's instructions. Cells were transfected for 48 hours before harvested, the RhebsiRNA efficiency was confirmed by Western blotting analysis. 


\section{Cellular Physiology Cell Physiol Biochem 2017;42:2481-2491

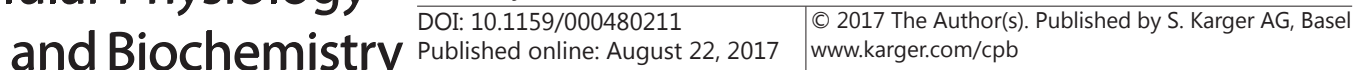 \\ Wang et al.: MicroRNA-199a-5p Inhibits Ankylosing Spondylitis Pathogenesis}

Rheb plasmid construction and cell transfection

According to the manufacturer's instructions, total RNA was isolated from the T cells by using TRIzol reagent (Invitrogen). The cDNA was synthesized with a PrimeScript RT reagent kit (Takara, Dalian, China) and the open reading frame of Rheb cDNA was cloned and inserted into the pcDNA3.1 vector (Invitrogen). Negative control cells were transfected with pcDNA3.1 vector alone. The cells were cultured to $60 \%$ confluence before transfection, and transfected by the FuGENE HD transfection reagent (Roche).

Cell viability assay

Cells were incubated $48 \mathrm{~h}$ post-transfection with MTT solution $(0.5 \mathrm{mg} / \mathrm{ml})$ at $37^{\circ} \mathrm{C}$ for $4 \mathrm{~h}$, then washed with PBS three times dimethyl sulfoxide (DMSO) was used to dissolve the insoluble formazan product. Cell viability was determined by the absorbance at $490 \mathrm{~nm}$ using a microplate reader.

In silico prediction of miR-199a-5p targets

The EIMMomiRNA target prediction server (http://www.mirz.unibas.ch/ElMMo3), microRNA.org (http://www.microrna.org), and TargetScan 5.1 (http://www.targetscan.org) were used to predict targets of miR-199a-5p.

Luciferase reporter assay

The Rheb 3'-untranslated region (3'-UTR) containing the miR-199a-5p predicted binding site (pGLORheb-BS) and Rheb 3'UTR with a mutated seed region for the predicted miR-199a-5p binding site (pGLORheb-Mut) were cloned into the 3'-UTR of the firefly luciferase structural gene in the pmirGLO DualLuciferase miRNA Target Expression Vector (Promega, Madison, WI, USA), respectively. HEK293 cells from a human embryonic kidney cell line were plated in 24 -well plates at a density of $1.5 \times 10^{5}$ cells per well in triplicate. After $24 \mathrm{~h}$, the cells were transfected with100 ng of the respective plasmid and $100 \mathrm{nmol} / \mathrm{L} \mathrm{miR}$ 199a-5p or control mimic using Lipofectamine 2000. Luciferase activities were measured $48 \mathrm{~h}$ after plasmid vector transfection using the dual luciferase reporter assay kit (Promega) following the manufacturer's instructions.

\section{Statistical analysis}

The results obtained in this study are expressed as mean value \pm standard deviation (SD) (quantitative variables) or as frequencies and percentages (qualitative variables). Differences between two groups with quantitative variables were assessed with the independent samples $t$-test and the Tukey-Kramer test after a significant one-way analysis of variance F-test for the multiple-group comparisons. Qualitative variables were assessed with the Mann-Whitney U test for nonparametric data. Correlation analyses were carried out using Pearson's rank correlation test, and $\mathrm{p}<0.05$ was considered statistically significant. All statistical analyses were conducted using Statistical Package for Social Sciences 13.0 software for Windows.

\section{Results}

\section{Subject characteristics}

Table 1 summarized the demographic and clinical characteristics of the study subjects. There were no differences between mean age or gender of AS patients and healthy controls. The disease duration of AS was $22.4 \pm 13.9$ months, mean ASDAS was $3.1 \pm 1.4$, and mean mSASSS was $32 \pm 13$. At the time of study enrollment, 38 patients were receiving non-steroid anti-inflammatory drugs (NSAIDs) therapy, and 7 were taking disease-modifying antirheumatic drugs (DMARDs).

Decreased miR-199a-5p expression in $T$ cells inhibited autophagy, and increased radiographic severity in ankylosing spondylitis patients

RT-PCR analysis showed that the miR-199a-5p expression was significantly lower in AS patients compared to healthy controls $(0.56 \pm 0.17 v s .1 .35 \pm 0.35, p<0.0001$; Fig. $1 \mathrm{~A})$. Simultaneously, expression of autophagy-related genes LC3, beclin1, and ATG5 mRNA in PBMCs was significantly down-regulated in AS patients, compared with the control group 
Table 1. Demographic and clinical characteristics of the study population. Data are presented as number or mean \pm standard deviation. AS, ankylosing spondylitis; ASDAS, ankylosing spondylitis disease activity score; mSASSS, modified stoke ankylosing spondylitis spine score; NASAIDs, non-steroidal anti-inflammatory drugs; DMARDs, disease-modifying anti-rheumatic drugs; IBD, inflammatory bowel disease; ND not determined; $n$, number of patients

\begin{tabular}{llccc}
\hline Characteristics & AS $(\mathrm{n}=41)$ & $\begin{array}{c}\text { Healthy controls } \\
(\mathrm{n}=36)\end{array}$ & $t / \chi^{2}$ & $p$ \\
\hline Age (years) & $33.7 \pm 12.4$ & $31.4 \pm 10.2$ & -0.881 & 0.381 \\
Gender, male/female (n) & $30 / 11$ & $20 / 16$ & 2.612 & 0.106 \\
Disease duration (months) & $22.4 \pm 13.9$ & - & & ND \\
HLA-B27 positive (n) & 41 & - & & ND \\
ASDAS & $3.1 \pm 1.4$ & - & & ND \\
mSASSS & $32 \pm 13$ & - & & ND \\
Use of NASAIDs & 38 & - & & ND \\
Use of DMARDs & 7 & - & & ND \\
Presence of IBD & 0 & - &
\end{tabular}

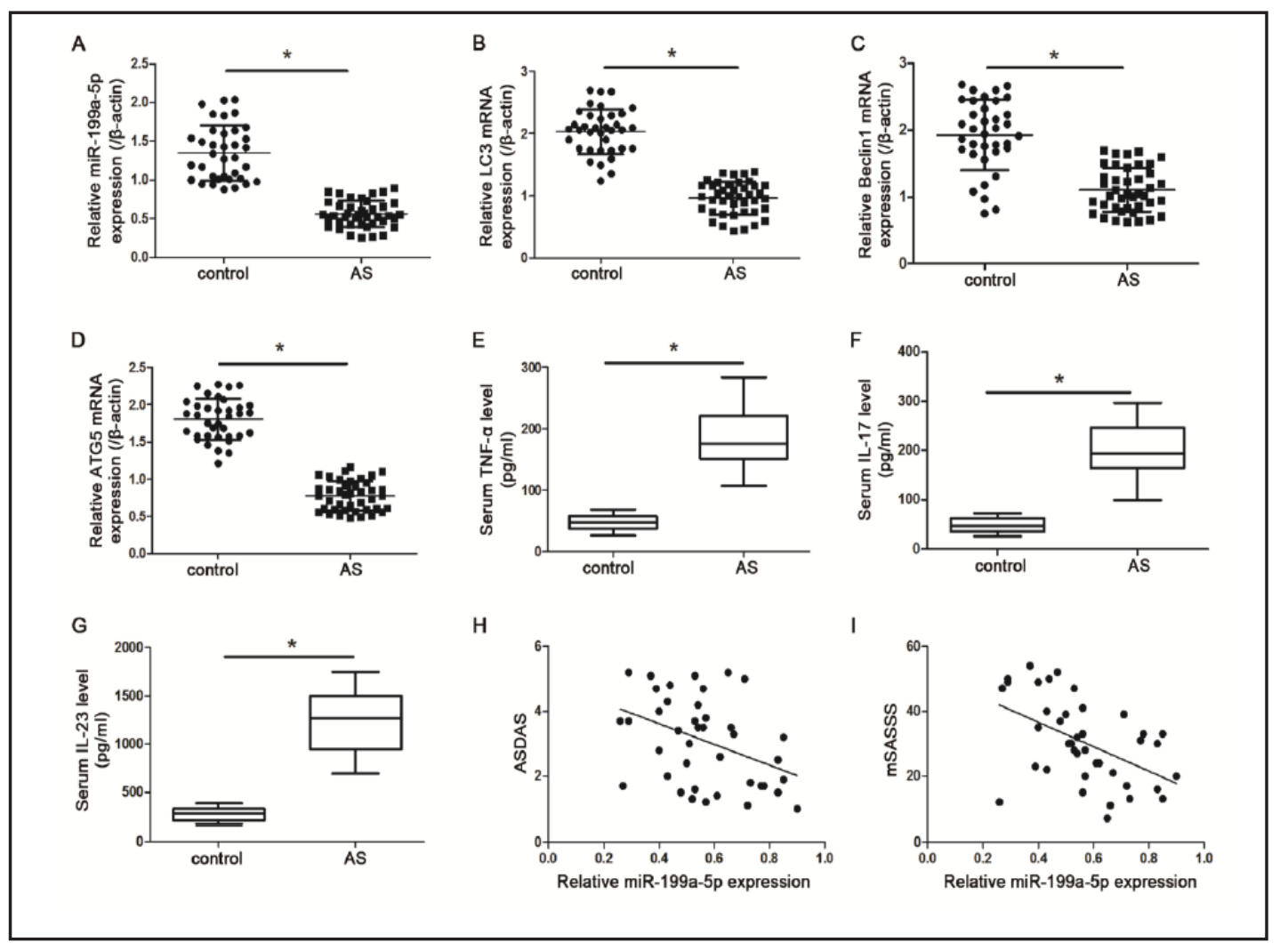

Fig. 1. MiR-199a-5p was down-regulated in T cells from ankylosing spondylitis patients, accompanied by inhibited autophagy level and increased radiographic severity. The mRNA expression of miR-199a-5p (A) and autophagy-related gene LC3 (B), beclin1 (C), and ATG5 (D) from the T cells of AS patients and controls were assessed by RT-PCR. Serum concentrations of TNF- $\alpha$ (E), IL-17 (F) and IL-23 (G) of AS patients and controls were detected by ELISA kits. Relationships between miR-199a-5p expression level and the Ankylosing Spondylitis Disease Activity Score (H) and the modified Stoke AS Spinal Score (I) were analyzed by Pearson's rank correlation test. Data are expressed as the mean \pm SD. ${ }^{*}$ Compared with the controls, $\mathrm{p}<0.05$.

$(0.96 \pm 0.26$ vs. $2.03 \pm 0.36,1.11 \pm 0.33$ vs. $1.93 \pm 0.52,0.77 \pm 0.20$ vs. $1.80 \pm 0.28, p<0.0001$, respectively; Fig. 1B, C, D). Consistent with characterization of AS as a chronic inflammatory disease, our results show that the levels of serum TNF- $\alpha$, IL-17 and IL-23, which are known 


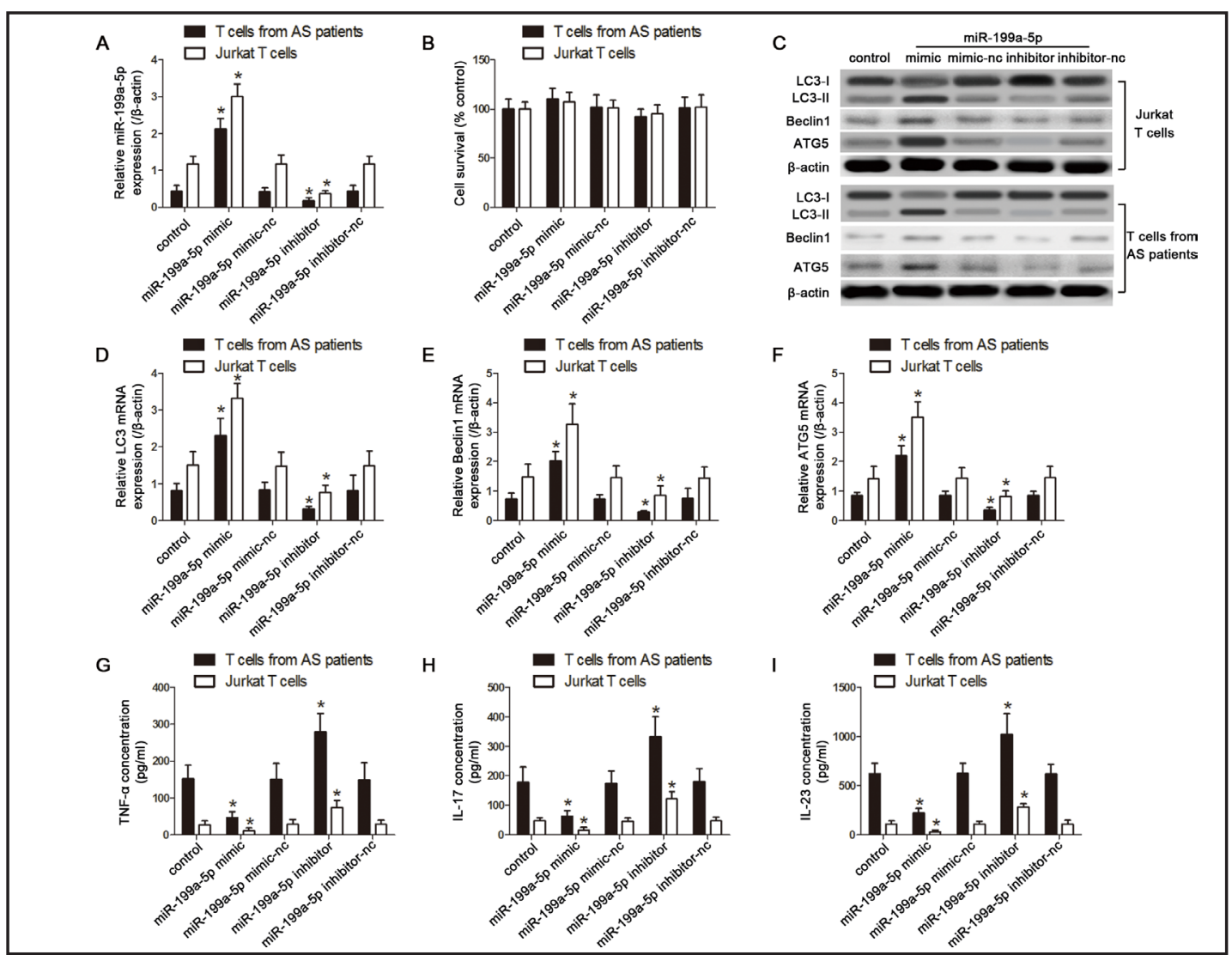

Fig. 2. Effect of miR-199a-5p on cell autophagy and pro-inflammatory cytokine production of Jurkat T cells and T cells from AS patients. MiR-199a-5p expression after transfection with miR-21 mimic or inhibitor (A). Detection of miR-199a-5p mimics and miR-199a-5p inhibitor on cell survival of Jurkat $\mathrm{T}$ cells and $\mathrm{T}$ cells from ankylosing spondylitis patients by MTT assay (B). The protein expression of autophagy-related genes was analyzed by Western blot after pretreatment with miR-199a-5p mimics or miR-199a-5p inhibitor for 48h (C). After pretreatment with miR-199a-5p mimics or miR-199a-5p inhibitor, miRNA levels of LC3 (D), beclin1 (E), and ATG5 (F) were analyzed by qRT-PCR. Pro-inflammatory cytokine TNF- $\alpha(G)$, IL-17 (H), and IL-23 (I) production was detected by ELISA kits. Data are expressed as the mean \pm SD.*Compared with corresponding controls, $\mathrm{p}<0.05$.

inflammatory biomarkers, were significantly higher in AS patients than in healthy controls (all $p<0.0001$; Fig. 1E, F and G).

ASDAS and mSASSS are general assessments of the activity and severity of AS. Univariate correlation analysis showed that the mRNA expression levels of miR-199a-5p were negatively correlated with the ASDAS and mSASSS $\left(r^{2}=0.1600, \mathrm{p}=0.0096 ; r^{2}=0.2503, \mathrm{p}=0.0009\right)$ (Fig. 1H and I). These data suggest that miR-199a-5p may influence cell autophagy and development of AS, reflecting disease activity and functional ability in AS patients.

MiR-199a-5p regulated autophagy and pro-inflammatory cytokine production in Jurkat T cells and $T$ cells from AS patients

To explore the effect of miR-199a-5p on pathogenesis of AS, Jurkat T cells and T cells isolated from PBMCs of AS patients were transfected with miR-199a-5p mimics, miR-199a$5 p$ inhibitor, or miR controls (mimic-nc/inhibitor-nc). Both the miR-199a-5p mimics and inhibitor were successfully transfected in the two cell lines (Fig. 2A). The MTT assay showed no difference between the treatment group and controls (Fig. 2B).

The assay of autophagy-related genes demonstrated that compared with the control group, the protein levels of LC3-II, beclin1, and ATG5 in both Jurkat T cells and AS patient 


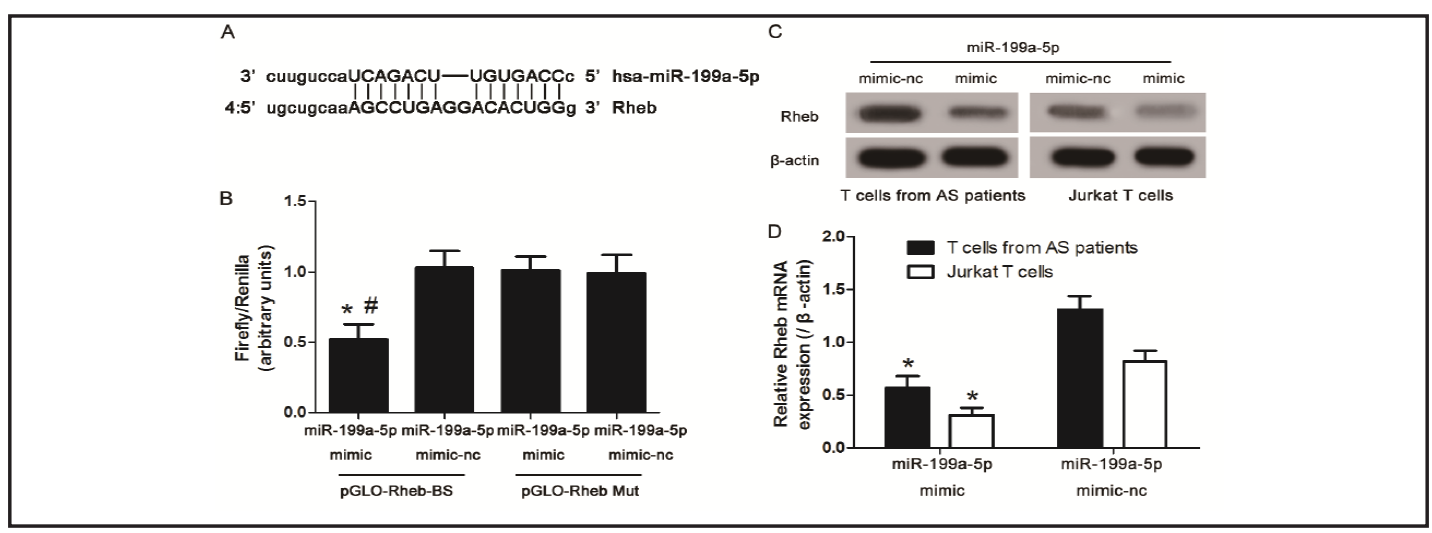

Fig. 3. Rheb is a direct target gene of miR-199a-5p. The predicted binding sequences of Rheb and miR-199a$5 p(A)$. The luciferase activity was measured using the dual-luciferase reporter assay following transfection with a plasmid containing a Rheb $3^{\prime}$-UTR binding site (pGLO-Prep-BS), or a mutated binding site (pGLOPrep-Mut) (B). Jurkat T cells and T cells from ankylosing spondylitis patients were transfected with miR199a-5p mimic and showed significant reductions in Rheb protein (C) and mRNA (D) expression. *Compared with pGLO-Prep-BS (miR-199a-5p mimic-nc), p < 0.05. \# Compared with pGLO-Prep-Mut (miR-199a-5p mimic), $\mathrm{p}<0.05$.

T cells were up-regulated after transfection with miR-199a-5p mimics (Fig. 2C). The LC3II, beclin1, and ATG5 mRNA levels also increased upon miR-199a-5p over-expression, in accordance with the protein data. Indeed, transfection of the miR-199a-5p mimic into the T cells induced an approximately 3 -fold increase in the level of LC3-II/I mRNA (Fig. 2D), a 2.5-fold increase in the level of beclin1 (Fig. 2E) and a 3-fold increase in the level of ATG5 (Fig. 2F), whereas TNF- $\alpha$, IL-17 and IL-23 were significantly reduced (p < 0.05) (Fig. G-I). Conversely, inhibition of miR-199a-5p showed the opposite effects. After transfection with the miR-199a-5p inhibitor, expression levels of LC3-II, beclin1, and ATG5 were downregulated, but TNF- $\alpha$, IL-17 and IL-23 increased.

\section{MiR-199a-5p directly interacted with the 3'UTR of Rheb}

Rheb is a high-ranked target gene of miR-199a-5p in silico, and has been reported to be involved in cell autophagy. The binding of miR-199a-5p with the 3'-UTR was shown in Fig. 3A. A luciferase reporter assay showed that the HEK293 cells transfected with pGLOHMGB1-BS plasmid and the miR-199a-5p mimic had an approximately $60 \%$ reduction of luciferase activity, compared with the controls. Mutation of the target site prevented the down-regulation of luciferase activity that is induced by miR-199a-5p (Fig. 3B). Moreover, the target relationship also was confirmed in both Jurkat T cells and AS patient T cells, Western blot and RT-PCR showed that miR-199a-5p mimic transfection can significantly reduce Rheb in protein (Fig. 3C) and mRNA (Fig. 3D).

\section{Rheb silencing contributed to T cell autophagy and decreased pro-inflammatory cytokine production}

To determine whether Rheb influenced T cell autophagy and AS pathogenesis, we transfected both Jurkat T cells and the T cells from AS patients with RhebsiRNA and pcDNA3.1Rheb (Fig. 4A). Results showed that RhebsiRNA significantly promoted the expression of LC3-II/I, beclin1 and ATG5 in both mRNA (Fig. 4B-D) and protein (Fig. 4E). It significantly reduced the release of TNF- $\alpha$, IL-17 and IL-23 (Fig. 4F-H) in the treatment groups compared with the control group. Moreover, Rheb silence also decreased the phosphorylation level of mTOR, and promoted ATG1 expression (Fig. 4I). Conversely, pcDNA3.1-Rheb transfection displayed the opposite effects. Thus, we speculate that miR-199a-5p regulates $\mathrm{T}$ cell autophagy and pro-inflammatory cytokine production by suppressing Rheb expression and inhibiting activation of the mTOR pathway.

\section{KARGER}




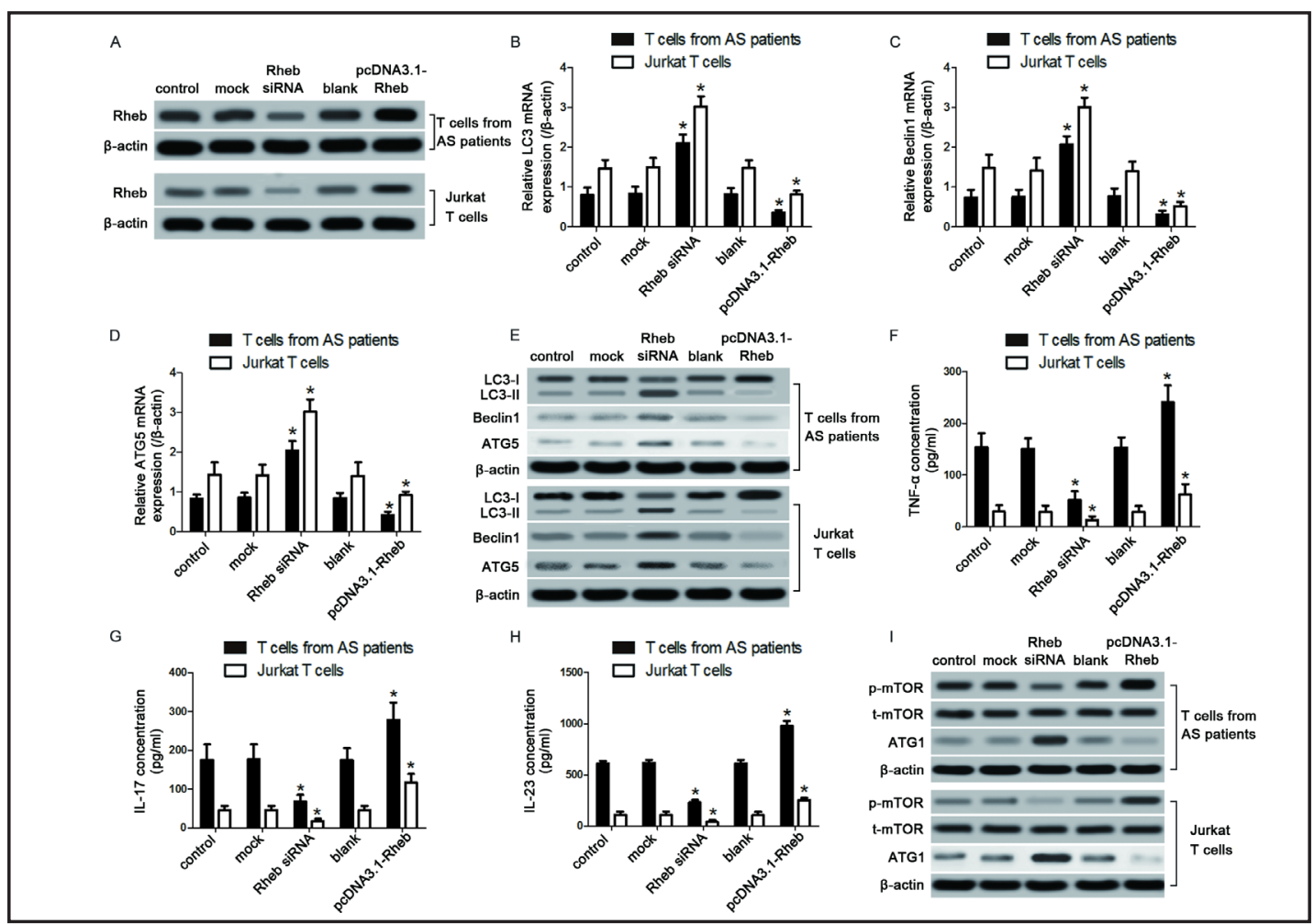

Fig. 4. Rheb expression regulated T cell autophagy and pro-inflammatory cytokine production. After pretreatment of Jurkat $\mathrm{T}$ cells and T cells from ankylosing spondylitis patients with Rheb siRNA or pcDNA3.1-Rheb for $48 \mathrm{~h}$, Rheb protein expression was detected by Western blot (A). The mRNA expression of autophagy-related genes LC3 (B), beclin1 (C), and ATG5 (D) was analyzed by qRT-PCR. Corresponding protein (E) expression was analyzed by Western blot. Production of pro-inflammatory cytokines TNF- $\alpha$ (F), IL-17 (G), and IL-23 (H) was detected by ELISA kits. The effects of Rheb siRNA and pcDNA3.1-Rheb pretreatment on mTOR phosphorylation and ATG1 protein expression were also analyzed by Western blot. (I). * Compared with the corresponding control group, $\mathrm{p}<0.05$.

\section{Rheb inhibited T cell autophagy and promoted pro-inflammatory cytokine production by} activating mTOR signaling

The mTOR antagonist Rapamycin was applied in the medium for 24 hours for further analysis. As shown in Fig. 5A-D, Rheb overexpression significantly reduced LC3-II/I, beclin1, and ATG5 expression in both mRNA and protein. It also increased the concentrations of TNF- $\alpha$, IL-17 and IL-23 (Fig. 5E, F and G, respectively). However, Rapamycin pretreatment markedly increased mRNA and protein expression of LC3-II/I, beclin1 and ATG5, and it decreased TNF- $\alpha$, IL-17 and IL-23 production when compared with the pcDNA3.1-Rheb group. These findings suggest that Rheb may inhibit cell autophagy and promote pro-inflammatory cytokine production that is attenuated by mTOR signaling inhibition. Although, Rheb overexpression showed a trend towards inhibiting $\mathrm{T}$ cell survival, there was no significant difference compared with the control (Fig. 5H).

\section{Discussion}

In this study, we demonstrated a novel role for miR-199a-5p in regulating autophagy in the pathogenesis of AS by modulating the mTOR signaling though direct targeting of Rheb. We found that miR-199a-5p was down-regulated in T cells, accompanied by inhibited autophagy levels, increased pro-inflammatory cytokine release, and radiographic severity in AS patients, compared with the controls. Further mechanism analysis suggested that miR- 


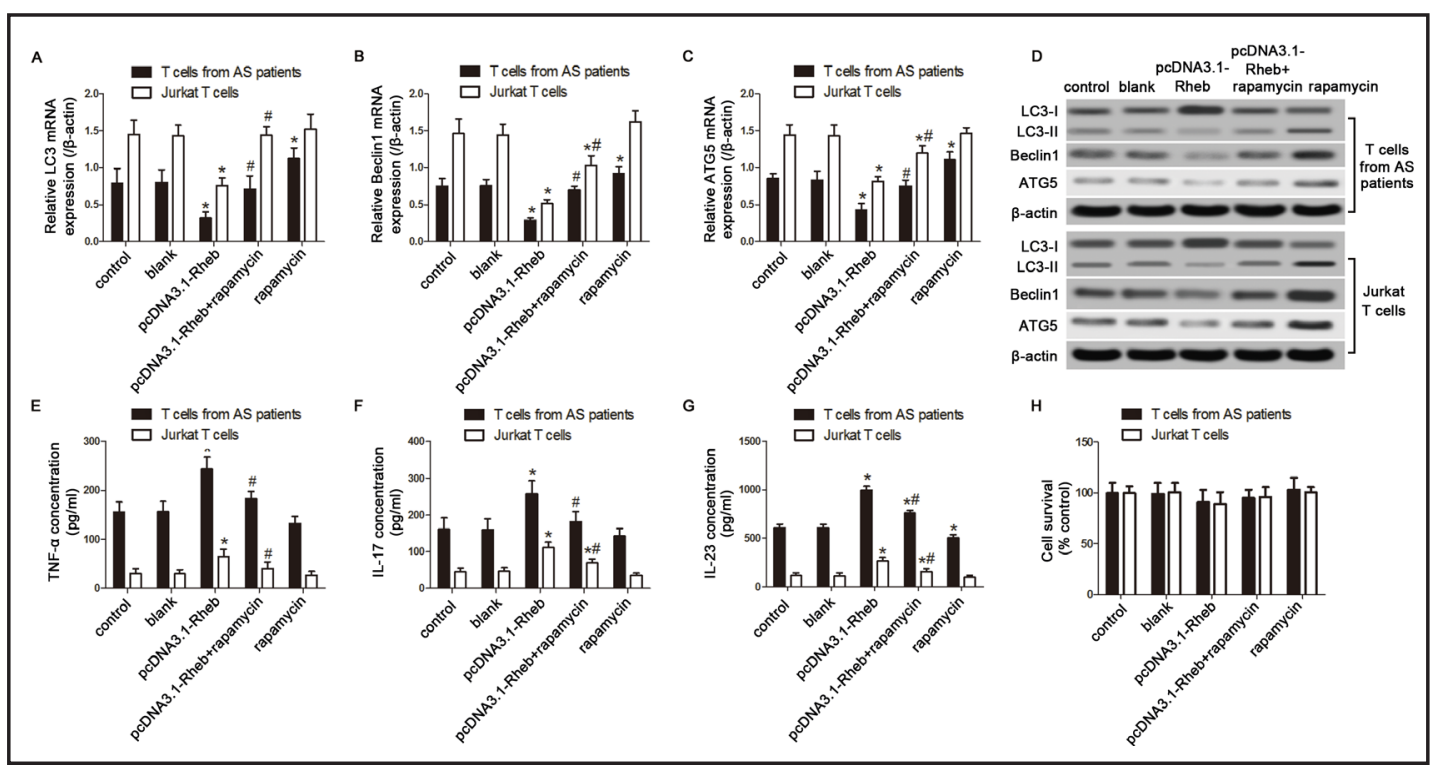

Fig. 5. Rheb inhibited $\mathrm{T}$ cell autophagy and promoted pro-inflammatory cytokine production by activating mTOR signaling. After pretreatment with the mTOR antagonist Rapamycin for $24 \mathrm{~h}$, mRNA expression of autophagy-related gene LC3 (A), beclin1 (B), and ATG5 (C) was analyzed by qRT-PCR. The corresponding protein (D) expression was analyzed by Western blot. Production of cytokine TNF- $\alpha$ (E), IL-17 (F) and IL-23 $(G)$ production was detected by ELISA kits. The survival of Jurkat T cells and T cells from ankylosing spondylitis patients was detected by MTT assay $(\mathrm{H}){ }^{*} \mathrm{p}<0.05$, compared with controls. \# $\mathrm{p}<0.05$, compared with the pcDNA3.1-Rheb group.

199a-5p regulated autophagy and pro-inflammatory cytokine production in both Jurkat $\mathrm{T}$ cells and T cells from AS patients by targeting Rheb. Rheb inhibited T cells autophagy and promotes pro-inflammatory cytokine production by activating mTOR signaling. These data suggest that miR-199a-5p participates in the regulation of AS pathogenesis by affecting $\mathrm{T}$ cell autophagy.

Autophagy removes pathogens and dead cells to maintain cellular homeostasis. Defects in this process may cause inflammation and exacerbate autoimmune disease [19]. Recent studies have shown that autophagy may play a critical role in the pathogenesis of AS [8]. LC3 executes autophagy, beclin 1 regulates autophagy, and ATG5 is involved in the maturation of autophagy. In the current study, LC3, beclin1, and ATG5 are all expressed in the T cells of AS patients, but at significantly decreased gene expression levels. These findings suggest that AS patients may have insufficient or defective autophagic activity. Defective autophagy can increase the inflammatory burden by failing to regulate the release of several proinflammatory cytokines, including TNF- $\alpha$, IL-4, IL-1 $\beta$, IL-17, and IL-23 [3, 20-23]. In this study, the serum levels of the pro-inflammatory cytokines TNF- $\alpha$, IL-17, and IL-23 were much higher in AS patients than in controls. These results are consistent with the notion that autophagy protects against inflammatory diseases and that defective autophagy contributes to the accumulation of damaged macromolecules and susceptibility to inflammation.

Dysregulation of miRNAs has been found to cause several pathological states by modulating cell autophagy [24]. According to previous studies, miR-222 helps to regulate of pathological cardiac remodeling and heart failure by mediating cardiomyocyte autophagy [25], miR-124 is involved autophagy in the peripheral blood from AS patients [26], and miRNA let-7i induces autophagy to protect $\mathrm{T}$ cells from apoptosis [27]. Our data show that miR-199a-5p was down-regulated in T cells from AS patients, and that the administration of miR-199a-5p mimics markedly enhanced the expression of autophagy-related genes. However, transfection of a miR-199a-5p inhibitor into both Jurkat T cells and T cells from AS patients suppressed autophagy levels by down-regulating the expression of related genes, and it promoted pro-inflammatory cytokine production. Interestingly, we found that miR- 


\section{Cellular Physiology Cell Physiol Biochem 2017;42:2481-2491 \begin{tabular}{l|l} 
DOI: 10.1159/000480211 22, 2017 & $\begin{array}{l}\text { O } 2017 \text { The Author(s). Published by S. Karger AG, Basel } \\
\text { www.karger.com/cpb }\end{array}$ \\
\cline { 2 - 3 } and Biochemistred online: August 22,
\end{tabular} \\ Wang et al.: MicroRNA-199a-5p Inhibits Ankylosing Spondylitis Pathogenesis}

199a-5p had little influence on T cell survival.

Most miRNAs carry out their biological function by binding to their target molecules [28]. To investigate how miR-199a-5p affects T cells autophagy, bioinformatics tools were used to search for potential targets of miR-199a-5p. Among the targets of miR-199a-5p, Rheb is an upstream positive regulator of mTOR signaling. Overexpression of Rheb in mammalian cells leads to mTORC1 activation [29]. Thus, we speculated that miR-199a-5p may influence T cell autophagy by modulating Rheb expression. In this study, increased miR-199a-5p expression reduced mRNA and protein levels of Rheb. Furthermore, we identified Rheb as a direct target of miR-199a-5p. Rheb siRNA significantly promoted LC3, beclin1 and ATG5 expression, and it suppressed TNF- $\alpha$, IL-17, and IL-23 production.

MTOR functions as a central sensor of cellular energy that responds to many metabolic events and controls autophagy [30,31]. It is a pivotal upstream mediator of autophagy through its binding and inactivation of the autophagy kinase complex ULK1/2, which blocks formation of autophagosomes [32]. To clarify whether mTOR signaling affected the suppression effects of Rheb in cell autophagy, the mTOR signaling antagonist Rapamycin was used. As predicted in our hypothesis, Rheb overexpression decreased ATG1 expression, and Rapamycin pretreatment could significantly attenuated the inhibitory effect on cell autophagy induced by Rheb.

Our data consistently showed that miR-199a-5p expression decreased in AS patients, was negatively correlated with disease severity and activity, and was accompanied by inhibited autophagy level. These findings indicate that down-regulation of miR-199a-5p and autophagy inhibition may be associated with AS pathogenesis. Furthermore, mechanistic analysis showed that Rheb contributed to T cell autophagy via the mTOR signaling pathway. Taken together, our data suggest that miR-199a-5p may regulate $T$ cell autophagy by the mTOR pathway via direct targeting of Rheb. Therefore, this study suggests that miR-199a-5p may be a potential therapeutic target for AS.

\section{Disclosure Statement}

The authors declared no conflicts of interest.

\section{References}

1 Fessler J, Raicht A, Husic R, Ficjan A, Duftner C, Schwinger W, Dejaco C, Schirmer M: Premature senescence of t-cell subsets in axial spondyloarthritis. Ann Rheum Dis 2016;75:748-754.

2 Yu Z, Zhang Y, Gao N, Yong K: Suppression of development of ankylosing spondylitis through soluble flt-1. Cell Physiol Biochem 2015;37:2135-2142.

- Z Zou YC, Yang XW, Yuan SG, Zhang P, Li YK: Celastrol inhibits prostaglandin e2-induced proliferation and osteogenic differentiation of fibroblasts isolated from ankylosing spondylitis hip tissues in vitro. Drug Des Devel Ther 2016;10:933-948.

4 Hou ZD, Xiao ZY, Gong Y, Zhang YP, Zeng QY: Arylamine n-acetyltransferase polymorphisms in han chinese patients with ankylosing spondylitis and their correlation to the adverse drug reactions to sulfasalazine. BMC Pharmacol Toxicol 2014;15:64-80.

5 Liu Z, Zhang P, Dong J: Genetic variants of stat4 are associated with ankylosing spondylitis susceptibility and severity in a chinese han population. Int J Clin Exp Med 2014;7:5877-5881.

6 Haywood KL, Packham JC, Jordan KP: Assessing fatigue in ankylosing spondylitis: The importance of frequency and severity. Rheumatology (Oxford) 2014;53:552-556.

7 Gan FY, Fei YY, Li MT, Wang Q Xu D, Hou Y, Zeng XF, Zhang FC: The characteristics of patients having ankylosing spondylitis associated with takayasu's arteritis. Clin Rheumatol 2014;33:355-358.

8 Ciccia F, Haroon N: Autophagy in the pathogenesis of ankylosing spondylitis. Clin Rheumatol 2016;35:1433-1436.

-9 Park MC, Kim HW, Lee SW, Song JJ, Park YB: Defective autophagy activity and its association with spinal damage in patients with ankylosing spondylitis. Joint Bone Spine DOI:10.1016/j.jbspin.2016.09.005. 


\section{Cellular Physiology Cell Physiol Biochem 2017;42:2481-2491 \begin{tabular}{c|c|c|} 
DOI: 10.1159/000480211 2017 & $\begin{array}{l}\text { O } 2017 \text { The Author(s). Published by S. Karger AG, Basel } \\
\text { www.karger.com/cpb }\end{array}$
\end{tabular} \\ Wang et al.: MicroRNA-199a-5p Inhibits Ankylosing Spondylitis Pathogenesis}

10 Xilouri M, Brekk OR, Polissidis A, Chrysanthou-Piterou M, Kloukina I, Stefanis L: Impairment of chaperonemediated autophagy induces dopaminergic neurodegeneration in rats. Autophagy 2016;12:2230-2247.

11 Pan Z, Zhao Y, Yu H, Liu D, Xu H: [effect of hydrogen-rich saline on cardiomyocyte autophagy during myocardial ischemia-reperfusion in aged rats]. Zhonghua Yi Xue Za Zhi 2015;95:2022-2026.

12 Chapman CG, Pekow J: The emerging role of mirnas in inflammatory bowel disease: A review. Therap Adv Gastroenterol 2015;8:4-22.

13 Neerinckx B, Carter S, Lories R: Il-23 expression and activation of autophagy in synovium and pbmcs of hla-b27 positive patients with ankylosing spondylitis. Response to: 'Evidence that autophagy, but not the unfolded protein response, regulates the expression of il-23 in the gut of patients with ankylosing spondylitis and subclinical gut inflammation' by ciccia et al. Ann Rheum Dis DOI:10.1136/ annrheumdis-2014-206277.

14 Gaur N, Karouzakis E, Gluck S, Bagdonas E, Jungel A, Michel BA, Gay RE, Gay S, Frank-Bertoncelj M, Neidhart M: Micrornas interfere with DNA methylation in rheumatoid arthritis synovial fibroblasts. RMD Open DOI:10.1136/rmdopen-2016-000299.

15 Freiesleben S, Hecker M, Zettl UK, Fuellen G, Taher L: Analysis of microrna and gene expression profiles in multiple sclerosis: Integrating interaction data to uncover regulatory mechanisms. Sci Rep DOI:10.1038/ srep34512.

16 Jing Z, Han W, Sui X, Xie J, Pan H: Interaction of autophagy with micrornas and their potential therapeutic implications in human cancers. Cancer Lett 2015;356:332-338.

17 Li Z, Wong SH, Shen J, Chan MT, Wu WK: The role of micrornas in ankylosing spondylitis. Medicine (Baltimore) DOI:10.1097/MD.0000000000003325.

18 van der Linden S, Valkenburg HA, Cats A: Evaluation of diagnostic criteria for ankylosing spondylitis. A proposal for modification of the new york criteria. Arthritis \& Rheumatism 1984;27:361-368.

19 Bhattacharya A, Eissa NT: Autophagy and autoimmunity crosstalks. Front Immunol 2013;4:88-110.

-20 Ciccia F, Accardo-Palumbo A, Rizzo A, Guggino G, Raimondo S, Giardina A, Cannizzaro A, Colbert RA, Alessandro R, Triolo G: Evidence that autophagy, but not the unfolded protein response, regulates the expression of il-23 in the gut of patients with ankylosing spondylitis and subclinical gut inflammation. Ann Rheum Dis 2014;73:1566-1574.

21 Wendling D: Interleukin-17 targeted therapies in axial spondyloarthritis. Immunotherapy 2015;7:11251128.

22 Lin S, Qiu M, Chen J: Il-4 modulates macrophage polarization in ankylosing spondylitis. Cell Physiol Biochem 2015;35:2213-2222.

23 Wang Y, Wang H, Jiang J, Zhao D, Liu Y: Comparative efficacy and acceptability of anti-tnf-alpha therapy in ankylosing spondylitis: A mixed-treatments comparison. Cell Physiol Biochem 2016;39:1679-1694.

24 Liu N, Jiang N, Guo R, Jiang W, He Q-M, Xu Y-F, Li Y-Q, Tang L-L, Mao Y-P, Sun Y: Mir-451 inhibits cell growth and invasion by targeting mif and is associated with survival in nasopharyngeal carcinoma. Mol Cancer 2013;12:123-140.

25 Su M, Chen Z, Wang C, Song L, Zou Y, Zhang L, Hui R, Wang J: Cardiac-specific overexpression of mir-222 induces heart failure and inhibits autophagy in mice. Cell Physiol Biochem 2016;39:1503-1511.

26 Qin Z, Wang PY, Su DF, Liu X: Mirna-124 in immune system and immune disorders. Front Immunol 2016;7:406-425.

27 Hou C, Zhu M, Sun M, Lin Y: Microrna let-7i induced autophagy to protect t cell from apoptosis by targeting igf1r. Biochem Biophys Res Commun 2014;453:728-734.

28 Ebert MS, Sharp PA: Roles for micrornas in conferring robustness to biological processes. Cell 2012;149:515-524.

29 Mohan N, Shen Y, Dokmanovic M, Endo Y, Hirsch DS, Wu WJ: Vps34 regulates tsc1/tsc2 heterodimer to mediate rheb and mtorc1/s6k1 activation and cellular transformation. Oncotarget 2016;7:52239-52254.

30 Wan G, Xie W, Liu Z, Xu W, Lao Y, Huang N, Cui K, Liao M, He J, Jiang Y, Yang BB, Xu H, Xu N, Zhang Y: Hypoxia-induced mir155 is a potent autophagy inducer by targeting multiple players in the mtor pathway. Autophagy 2014;10:70-79.

-31 Carroll B, Maetzel D, Maddocks OD, Otten G, Ratcliff M, Smith GR, Dunlop EA, Passos JF, Davies OR, Jaenisch R, Tee AR, Sarkar S, Korolchuk VI: Control of tsc2-rheb signaling axis by arginine regulates mtorc1 activity. Elife DOI: 10.7554/eLife.11058.

32 Su M, Wang J, Wang C, Wang X, Dong W, Qiu W, Wang Y, Zhao X, Zou Y, Song L, Zhang L, Hui R: Microrna-221 inhibits autophagy and promotes heart failure by modulating the $\mathrm{p} 27 / \mathrm{cdk} 2 / \mathrm{mtor}$ axis. Cell Death Differ 2015;22:986-999. 